\title{
Influencia de los aspectos visuales en los cuestionarios autoadministrados
}

\author{
Vidal Díaz de Rada \\ Universidad Pública de Navarra. Departamento de Sociología \\ vidal@unavarra.es
}

Recepción: 03-05-2013

Aceptación: 13-02-2014

\section{Resumen}

El objetivo de este trabajo es analizar la influencia del diseño gráfico (tamaño del cuestionario, color del papel y existencia de portada y contraportada) en la tasa y en la calidad de la respuesta cuando se realizan encuestas postales. La calidad de la respuesta será definida considerando el número de preguntas no respondidas, la cantidad de respuestas en las preguntas multirrespuesta y la presencia del denominado «efecto primacía». Tras obtener una tasa de respuesta del 56,6\% (RR1), a lo largo del trabajo, se demuestra que ninguno de los elementos considerados afecta a la tasa ni a la calidad de la respuesta.

Palabras clave: encuestas por correo; tasa de respuesta; calidad de la respuesta; no respuesta parcial; efecto primacía; confección de cuestionarios.

\section{Abstract. Questionnaire layout and quality of response in self-administered surveys}

The aim of this paper is to analyze the influence of the size of the questionnaire, color of the paper, and the existence of front and back with a graphic design on the rate and quality of response in postal surveys. The quality of response is defined considering the number of unanswered questions, the number of answers in multiple-choice questions, and the presence of so-called primacy effect. After obtaining a response rate of $56.6 \%$ (RR1), the paper shows that none of the elements considered affect the rate and quality of response.

Keywords: postal and mail surveys; response rate; response quality; item non-response; primacy effect; questionnaire construction 


\author{
Sumario \\ Introducción 3. Resultados \\ 1. Aspectos visuales del cuestionario y \\ 4. Conclusiones \\ cambios en la respuesta: revisión de la \\ investigación internacional \\ Referencias bibliográficas \\ 2. Propósito y metodología \\ Anexo 1: Cuestionario utilizado
}

\title{
Introducción
}

Aunque un gran número de textos sobre investigación mediante encuesta insiste en la importancia del diseño del cuestionario en la tasa y en la calidad de la respuesta (entre otros, Sheastley, 1983; Manheim y Rich, 1986; Newman, 1997; Sudman y Bradburn, 1987; Czaja y Blair, 1996), hay escasas referencias a aspectos visuales como el tamaño del papel utilizado, el color y el diseño de la portada y la contraportada. Un análisis detallado de la literatura revela que la mayor parte de las recomendaciones se refiere a la presentación del encuestador, a la organización del cuestionario, al empleo de determinado tipo de preguntas, a la disposición y a las instrucciones para el entrevistador, a la numeración de las preguntas, al espacio que hay entre ellas, a la colocación de categorías de respuesta en sentido vertical u horizontal, etc. Hasta la última década del siglo $\mathrm{xx}$, los aspectos visuales del cuestionario eran importantes, porque se trataba del soporte donde el encuestador anotaba las respuestas, aunque, en la actualidad, desciende su protagonismo por la ausencia de "cuestionario físico» en las encuestas telefónicas y el paulatino desplazamiento de los cuestionarios «presenciales de papel» a los digitales (por la implantación de los asistidos por ordenador, en adelante CAPI).

En los últimos años, se ha producido un gran desarrollo de la encuesta autoadministrada (Couper, 2005, 2011), que ha generado una gran atención hacia los aspectos visuales del cuestionario ${ }^{1}$. Aunque muchas de estas investigaciones se han centrado en las encuestas a través de la red, diversos trabajos han demostrado que los errores que se encuentran en ellas descienden notablemente cuando se emplea una notificación postal para realizar la captación y la selección del entrevistado $^{2}$. Acceder al entrevistado a través del correo ordinario no sólo reduce el error de cobertura, sino que también aumenta la tasa de respuesta, al ofrecer la posibilidad de contestar el cuestionario de papel (incluido en la carta de presentación de la investigación) o mediante el acceso a un enlace donde se encuentran las preguntas. Existe un acuerdo unánime en considerar que la encuesta de papel presenta una mayor tasa de respuesta que la encuesta web (Kaplowitz et

1. Entre otros: Medway y Tourangeau, 2011; Messer y Dillman, 2011; Smyth y Olson, 2011; Stern et al., 2012; Sun, 2011; Tourangeau et al., 2004.

2. Bradley, 1999; Dillman et al., 2009; Heerwegh y Loosveldt, 2008; Heerwegh, 2009; Holmberg et al., 2010; Muñoz Leiva et al., 2009). 
al., 2004; Lozar Manfreda et al., 2008; Messer y Dillman, 2011; Medway y Fulton, 2012), lo que precisa prestar atención a todos los elementos que forman parte de este primer contacto, incluidos los visuales.

Resulta sorprendente la ausencia, en la literatura especializada, de las consideraciones sobre las dimensiones del cuestionario, el diseño de la portada y la contraportada y el color del papel utilizado. De hecho, y aunque algunos trabajos han demostrado la importancia del diseño gráfico del cuestionario en las entrevistas cara a cara (Sánchez, 1992), es en las encuestas autoadministradas donde la importancia es mayor. En la medida en que el cuestionario se convierte en la "carta de presentación» del investigador, se trata del único vínculo existente entre el que realiza la demanda de cooperación y el entrevistado, el «propietario» de la información.

En este trabajo, se analiza la influencia de determinados aspectos visuales del cuestionario en la tasa y en la calidad de respuesta, considerando concretamente el tamaño del cuestionario, el color de papel empleado, así como la existencia de portada y contraportada con un diseño gráfico.

\section{Aspectos visuales del cuestionario y cambios en la respuesta: revisión de la investigación internacional}

Una revisión exhaustiva de las publicaciones sobre el tema desvela el escaso número de investigaciones dedicadas a analizar cómo las dimensiones del cuestionario influyen en la tasa y en la calidad de las respuestas. En la obra más conocida sobre el tema, Dillman (1978) aconseja diseñar el cuestionario como un «folleto» de dimensiones 15,56 x 20,95 centímetros (en adelante, $\mathrm{cm}$ ) y con una grapa en el doble, aunque en ningún momento justifica los motivos de dicha decisión. En una obra posterior, Dillman (1991) considera que este aspecto de su Método del diseño total (Total Design Method, en adelante TDM) ha sido analizado en escasas ocasiones y anima a realizar más estudios sobre el tema.

En un estudio a población en general, Johnson et al. (1993) comparan un cuestionario de $14,5 \mathrm{~cm} \times 21,0 \mathrm{~cm}$ con otro de dimensiones $21,6 \mathrm{~cm} \times 27,9 \mathrm{~cm}$ (papel estándar en Estados Unidos). El segundo logró una tasa de cooperación del $64 \%$, un $61 \%$ de cuestionarios completos en su totalidad y un 2,6\% de preguntas sin responder; cifras que, en el cuestionario de $14,5 \mathrm{~cm} \times 21 \mathrm{~cm}$, descienden al 58\% (tasa de cooperación) y al 51\% (cuestionarios completos). El porcentaje de preguntas sin responder casi se duplica, puesto que llega al 4,3\% (Johnson et al., 1993). Sintetizando, el tamaño $21,6 \mathrm{~cm} \times 27,9 \mathrm{~cm}$ consigue aumentar la tasa de respuesta en seis puntos porcentuales, un menor número de preguntas sin contestar (casi la mitad) y un mayor porcentaje de cuestionarios completos (un 10\% más que en el otro tamaño). El análisis de estos resultados y de otros llevan a Dillman (1991) a plantear que los cuestionarios de dimensiones reducidas serán respondidos mejor por los jóvenes, mientras que la población de más edad preferirá cuestionarios mayores. En una obra posterior, Dillman (2000) recomienda cuestionarios de $21,6 \mathrm{~cm} \times 27,94 \mathrm{~cm}$, en base a los estudios realizados por otros investigadores de la sociedad norteamericana. 
Otro de los aspectos que Dillman considera muy importantes es el diseño del cuestionario en relación con la portada y la contraportada del mismo, puesto que es lo primero que observa el entrevistado y que condiciona su conducta (Dillman, 1978; Jenkins y Dillman, 1997). Dillman recomienda no realizar preguntas en la primera página. Esta debe utilizarse para poner un título interesante, un dibujo o cualquier otro elemento que llame la atención. Bajo este dibujo, puede ponerse una explicación sobre la investigación, un mensaje que señale la importancia de las opiniones del entrevistado e incluso instrucciones sobre cómo rellenar el cuestionario. También es conveniente colocar, a pie de página, el nombre y la dirección de la institución que promueve la investigación.

Entre los escasos estudios que han analizado la influencia de este aspecto, destaca el trabajo de Nederhof (1988), que demuestra que colocar un dibujo en la portada del cuestionario incrementa su tasa de respuesta, al aumentar la importancia que se le atribuye. Nederhof compara un mismo diseño de portada con fondo blanco (impresión en negro) y con fondo negro (impresión en blanco), y este último consigue una tasa de respuesta 15 puntos porcentuales superior al primero. Aunque los recordatorios sucesivos también incrementan la importancia del cuestionario, la impresión producida por la portada de fondo negro genera, en los entrevistados, un aumento de la importancia de la investigación (Nederhof, 1988). Ahora bien, unos años más tarde, Dillman y Dillman (1995) logran resultados opuestos en un estudio a la población general de Washington. Estas diferencias se atribuyeron al hecho de que el análisis de Nederhof se había realizado a una población especializada en su lugar de trabajo, por lo que podría estar más motivada que la población en general.

Respecto al color del cuestionario, y aunque Dillman (1978) señala que, para evitar el parecido con cualquier tipo de publicidad, este debe estar impreso en papel blanco, varios expertos han analizado cómo el color del cuestionario influye en la respuesta y, pese a que su uso puede recordar a los entrevistados el correo masivo o de baja calidad y ser contraproducente (Dillman, 1978), algunos estudios han indicado que sí influye en la tasa de respuesta. Un análisis de la literatura desvela que la comparación entre papel blanco y papel verde ha sido la más frecuente, y este último ha logrado un índice de contestación cinco puntos mayor (Fox et al., 1988). Similares resultados consigue Matteson (1974) utilizando un cuestionario rosa entre los miembros de una organización profesional, aunque otras investigaciones que utilizan el azul señalan que —aunque consigue más respuestas que el blanco- las diferencias no llegan a ser significativas (Jobber y Sanderson, 1983).

Entre las interpretaciones sobre dicha influencia, puede señalarse que la utilización de hojas de colores añade al cuestionario un cierto toque de conspicuidad cuando este se coloca en una mesa entre papeles blancos (Jobber y Sanderson, 1983), mientras que otras explicaciones están relacionadas con los efectos que cada uno de los colores utilizados causa en la mente de los entrevistados, que no serán citados aquí por estar fuera de los objetivos del trabajo (verde: esperanza, rojo: pasión, etc.). 


\section{Propósito y metodología}

Con el fin de comprobar hasta qué punto estos aspectos afectan a la tasa y a la calidad de las respuestas, los tres serán combinados en un cuestionario y comparados en una muestra dividida en partes equivalentes (split ballot). Es una estrategia metodológica (Petersen, 2008) muy utilizada en investigación con encuestas (entre otros, Schuman y Presser, 1981) y que consiste, básicamente, en dividir una muestra en dos partes equivalentes para aplicarles un cuestionario a ambas de forma simultánea y bajo las mismas condiciones. Se trata de un cuestionario que tiene «dos versiones» (por ejemplo: cambio en las palabras de una misma pregunta, cambio en el orden de las preguntas, etc.) y cada grupo responde una versión distinta. El planteamiento es que las diferencias entre los grupos, al tratarse de muestras equivalentes, serán producidas por los cambios en el cuestionario. En el apartado 2.a, se explica de forma pormenorizada cada uno de los elementos contemplados.

Preocupados por la posible influencia del tema del cuestionario en la respuesta (Groves et al., 2004), se optó, de todas las temáticas posibles, por un cuestionario sobre hábitos de consumo, un argumento muy habitual en estudios con encuestas. El cuestionario está formado por veintiséis preguntas, de las que cuatro son multirrespuestas categóricas (sin ninguna limitación con el número de respuestas), una multirrespuesta que limita el número de respuestas a dos, una pregunta de batería (con siete ítems y dos categorías de respuesta) y el resto se trata de preguntas tipo «abanico» o de "respuesta forzada» (forced choice), la mayoría con tres y cuatro categorías de contestación. Concretamente, siete presentan tres opciones de respuesta, cuatro preguntas tienen cuatro categorías y otras tantas, cinco ${ }^{3}$. Las siete preguntas sociodemográficas utilizadas recogen información sobre la relación con el cabeza de familia, el nivel de estudios, el estado civil, el sexo, la ocupación, el número de personas que residen en el hogar y la edad. Estas tres últimas emplean un formato abierto ${ }^{4}$. La ocupación fue codificada posteriormente, no así la edad ni el número de personas residentes en el hogar, que fueron tratadas como variables numéricas. Es importante destacar que el cuestionario fue elaborado siguiendo los criterios establecidos por Dillman (2000) en los capítulos 2, 3 y 4 de su obra.

El cuestionario fue administrado a una muestra representativa de la población Navarra, con direcciones extraídas de la guía telefónica. No obviamos que la gran cantidad de ausencias plantea dudas sobre la adecuación de utilizar este documento como marco muestral (Díaz de Rada y Andreu, 2004; Pasadas del Amo y Trujillo Carmona, 2013), aunque consideramos que su empleo es adecuado, con el fin de conocer cómo diferentes personas - pertenecientes a la

3. Detallamos el número de preguntas según las amplitudes de respuesta:

- Más de seis opciones: dos preguntas (P6a y P7a).

- Cinco opciones de respuesta: dos preguntas (P6 y P18).

- Cuatro opciones de respuesta: cinco preguntas (P1, P5, P15, P15a y P19).

- Tres opciones de respuesta: siete preguntas (P2, P3, P8, P9, P10, P11, P14).

- Dicotómicas: cuatro preguntas (P7, B9, P16b y P17).

4. En el anexo, se muestra el cuestionario utilizado. 
población general— reaccionan ante un cuestionario (Keeter, 1995; Tucker et al., 1992; Brick et al., 2012). La temática del cuestionario no presenta variaciones entre un tipo de población u otra, por lo que consideramos que, aunque esta prueba se haya realizado a una parte de la población — concretamente, las personas que tienen números listados (incluidos en la guía)—, el resto (la que haya «desaparecido» o nunca haya estado en tales guías) reaccionará de forma similar.

\subsection{Operacionalización de los elementos considerados}

Con el fin de comprobar si existen diferencias en la tasa y en la calidad de la respuesta en función de una serie de aspectos visuales, se confeccionaron ocho tipos de cuestionarios $(2 \times 2 \times 2)$ que fueron distribuidos aleatoriamente a la muestra seleccionada. Cada uno de estos cuestionarios presenta unas características propias en relación con distintos aspectos:

- Dimensiones: teniendo en cuenta la revisión de la literatura del primer apartado, la mitad de la muestra seleccionada recibió un cuestionario de $21 \mathrm{~cm} \times 29,7 \mathrm{~cm}$, muy similar al utilizado por Johnson et al. (1993). Conviene recordar que este es el tamaño recomendado por Dillman en uno de sus últimos libros (2000).

Se trata de una hoja tamaño DIN A-3 doblada por la mitad, que, a partir de este momento, recibirá el nombre de cuestionario grande. Las ligeras diferencias entre nuestro cuestionario y el utilizado por Johnson se basan en las que se encuentran entre los papeles habituales empleados en España y en Estados Unidos.

La otra mitad de la muestra recibió un cuestionario de $14,85 \mathrm{~cm} \times 21 \mathrm{~cm}$, similar al propuesto por Dillman en su Total Design Method (1978). Se trata de una hoja DIN A-4 doblada por el centro. A este tamaño, la mitad que el anterior, nos referiremos en el trabajo como cuestionario reducido.

- Formato de la portada y la contraportada: aspecto de gran importancia, por ser lo primero que observa una persona que recibe una comunicación escrita que no espera. La mitad no tenía ningún elemento en la portada ni en la contraportada. La primera página comenzaba con la primera pregunta del cuestionario, seguía en la segunda, la tercera y la cuarta, y finalizaba, en la parte final de la última página, con «Muchas gracias por su colaboración» (ver la parte final del anexo).

La otra mitad de los cuestionarios fueron diseñados siguiendo las recomendaciones de Dillman (1978). Había ausencia de preguntas en la primera página, que se reservaba para colocar elementos que llamaran la atención del receptor del cuestionario. En el centro de la portada, se colocó la silueta de la Comunidad Autónoma con la delimitación de cada uno de los municipios que la componían. Además, en cada cuestionario, se "pintó», con rotuladores de colores distintos, la silueta del municipio donde residía el entrevistado. La propia temática del cuestionario, hábitos de consumo, nos llevó a iniciar la página con un título que llamara la atención, una pregunta que pedía si eran diferentes los hábitos 
Figura 1. Portada del cuestionario

\section{¿SON DISTINTOS LOS HÁBITOS DE CONSUMO DE LOS NAVARROS? \\ UN ESTUDIO SOBRE HÁBITOS Y ACTITUDES DE CONSUMO \\ DE LA POBLACIÓN NAVARRA}

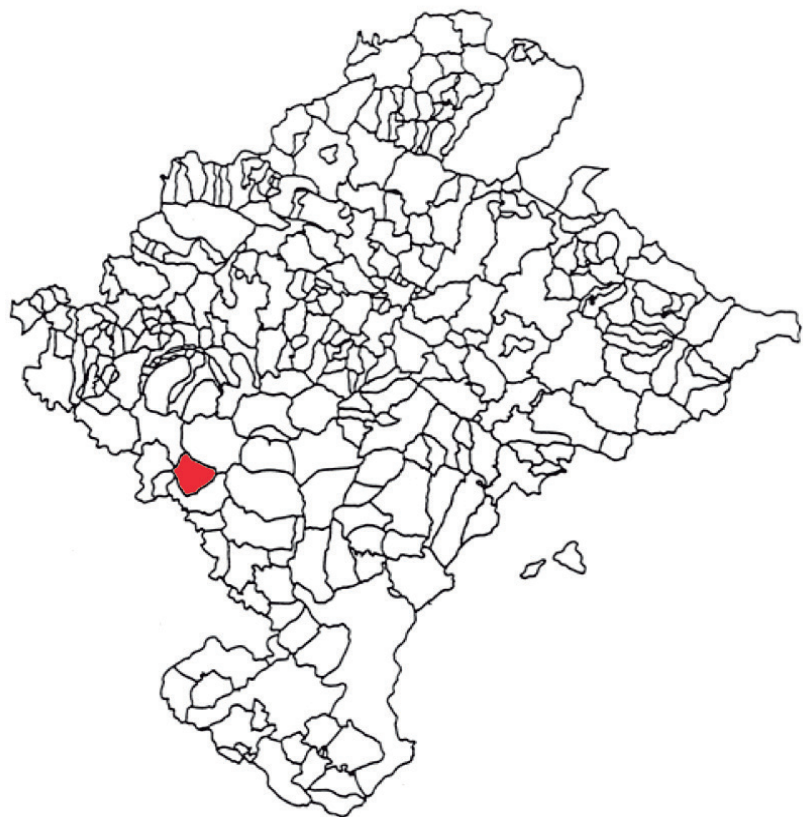

Muchísimas gracias por su colaboración.

Por favor, responda a todas las preguntas.

Puede utilizar el espacio de la parte de atrás de este cuaderno para anotar cualquier comentario.

Cuando esté terminado, introdúzcalo en el sobre adjunto (no necesita sello) y envíelo a:

\section{Universidad Pública de Navarra \\ Departamento de Sociología \\ Apartado n. ${ }^{\circ} 52$ F. D. \\ 31080 Pamplona \\ Tel.: 948168903}

Fuente: elaboración propia.

de consumo de los residentes en la comunidad navarra. La parte inferior de esta página se utilizó también para colocar instrucciones sobre la forma de proceder con el cuestionario e identificar la institución que realizaba el estudio.

Se contó también con un teléfono (que estuvo atendido desde las 9 de la mañana hasta las 9 de la noche) para aclarar posibles dudas. Aunque se solicitó uno sin coste (número 900), diversos problemas impidieron conseguirlo y se procedió a colocar el teléfono del director de la investigación. En la figura 1, se muestra un ejemplo de cuestionario enviado a Cárcar. 
Siguiendo las recomendaciones de Dillman, no se colocaron preguntas en la última página, donde se dejó espacio para recoger comentarios adicionales de los entrevistados (ver la última página del anexo).

- Color del cuestionario: la mitad de los cuestionarios fueron impresos en papel blanco, y el resto, en papel verde. Consideramos que este último obtendría más respuestas, debido a su mayor diferenciación.

Estas tres condiciones experimentales, con dos posibilidades cada una, generaron ocho tipos de cuestionarios $(2 \times 2 \times 2)$, cuya distribución se mostrará en el siguiente apartado dedicado al muestreo y al proceso para aumentar la tasa de respuesta.

\subsection{Diseño muestral}

Considerando que el objetivo no es representar adecuadamente a una población determinada, sino conocer el modo como una población general reacciona a los aspectos visuales del cuestionario, se estableció un tamaño mínimo de 50 entrevistas para la combinación de cada una de las situaciones experimentales anteriormente descritas. Los ocho grupos formados por la multiplicación de las tres condiciones experimentales con dos posibilidades cada una $(2 \times 2 \times 2)$ precisan de un tamaño muestral mínimo de 400 entrevistas $(50 \times 8)$.

Aunque el descenso progresivo en la tasa de respuestas en todos los países de nuestro entorno (entre otros, Benthlehem et al., 2011; Blom et al., 2008; Peytchev et al., 2009, Riba et al., 2010; Stoop et al., 2012) ha afectado menos a las encuestas autoadministradas (entre otros, De Leeuw et al., 2008; Kaplowitz et al., 2004), preferimos ser cautos a la hora de decidir el tamaño muestral y considerar — como punto de partida — que se obtendrá una tasa de respuesta reducida. La mayor parte de textos de investigación con encuesta cifra la tasa de respuesta de las encuestas postales entre un 30\% y un 50\% (entre otros, Sarantakos, 2003). En España, Visauta (1989: 285) sitúa esta tasa de respuesta entre el 20\% y el 70\%, mientras que otros trabajos más recientes dan cifras entre el 10\% y el 20\% (Ballina Ballina, 1998: 60), o entre el 10\% y el 15\% (Miquel et al., 1999: 69). En este caso, estamos pensando en un porcentaje de respuesta entre el $50 \%$ y el $55 \%$, y consideramos que es una tasa reducida, al compararla con investigaciones similares realizadas en el mismo contexto. Así, por ejemplo, encuestas realizadas por los autores en la sociedad navarra durante la última década del siglo xx y la primera del XXI han logrado tasas de respuesta comprendidas entre el 61,1\% y el $81,3 \% 5$ (Díaz de Rada, 1999; Díaz de Rada y Eguaras, 1999; Díaz de Rada, 2001).

5. Bien es verdad que esta tasa no es generalizable, al no tratarse de una población en general, sino de los socios de un club que fueron consultados por ese mismo club (Díaz de Rada y Eguaras, 1999).

Ahora bien, la gran involucración de la población entrevistada en la participación, unida al elevado número de comentarios en la pregunta situada en la contraportada del cuestionario, nos hizo dudar de la exactitud de la tasa de respuesta conseguida. El equipo investigador siempre ha creído que la tasa de participación fue superior, pero que, por problemas en el medio de distribución, no llegaron todos los cuestionarios respondidos. 
Tabla 1. Diseño muestral considerando las tres situaciones experimentales: muestra teórica

\begin{tabular}{llccc}
\hline & \multicolumn{2}{c}{ Diseño } & \\
\cline { 3 - 4 } & & Portada & No portada & Total \\
\hline Blanco & Tamaño pequeño & 98 & 98 & 196 \\
& Tamaño grande & 98 & 98 & 196 \\
& Total & 196 & 196 & 392 \\
\hline Verde & Tamaño pequeño & 98 & 98 & 196 \\
& Tamaño grande & 98 & 98 & 196 \\
& Total & 196 & 196 & 392 \\
\hline
\end{tabular}

Fuente: elaboración propia.

Considerar esta tasa de respuesta, junto con la necesidad de contar con 400 cuestionarios - 50 en cada situación experimental - recomendó un tamaño muestral inicial de 800 cuestionarios. El tamaño muestral definitivo quedó fijado en 784 (tabla 1). Los cuestionarios fueron distribuidos de forma proporcional considerando la comarca y el tamaño del municipio, enviando 247 cuestionarios a la capital, donde reside el 31,49\% de la población, 182 cuestionarios a municipios entre 10.001 y 50.000 habitantes (23,2\%), 97 a municipios entre 5.001 y 9.999 habitantes (12,3\% de la población), 145 a municipios entre 2.000 y 4.999 habitantes $(14,1 \%)$ y el resto $(114)$ a municipios menores de 5.000 habitantes. Aunque hubiera sido posible distribuir la muestra en todos los municipios navarros, porque el coste de la encuesta postal - a diferencia de la presencial - no varía cuando se aumenta la dispersión muestral, fueron seleccionados 20 municipios por motivos estratégicos, con el fin de tener la investigación más controlada.

Buscando conseguir la máxima respuesta, se utilizó un proceso de implementación similar al propuesto en el Tailored Design Method (Dillman, 2000), versión revisada del Total Design Method propuesto por Dillman a finales de la década de 1970 (1978) y que ha logrado tasas de respuesta elevadas en contextos muy diferentes ${ }^{6}$. El Tailored Design Method se fundamenta en la teoría del intercambio social (Dillman, 1978), que considera que cada persona tratará en todo momento de minimizar los costos de sus acciones y de aumentar las recompensas producidas por las mismas. Aplicado al ámbito que nos ocupa, un cuestionario tendrá más probabilidad de ser respondido cuando el esfuerzo para responderlo sea pequeño, la percepción de recibir una recompensa sea máxima y exista una confianza elevada en la recepción de tal recompensa.

6. Se han realizado investigaciones sobre su adecuación en Australia (Graetz, 1985); Estados Unidos (Dillman, 1991; Cook et al., 2000; Lozar Manfreda et al., 2008; Shih y Fan, 2008); Japón (Jussaume y Yamada, 1990); Rusia (Avdeyeva y Matland, 2013), y diversos países de Europa (Nederhof, 1988; De Leeuw, 1992; Díaz de Rada, 1999, 2000). 


\subsection{Delimitación de objetivos e hipótesis de trabajo}

El objetivo de la investigación es demostrar hasta qué punto los aspectos visuales del cuestionario afectan a la tasa y a la calidad de la respuesta en encuestas postales. Dentro de todos los factores posibles, en este trabajo, centraremos nuestra atención en la influencia de las dimensiones del cuestionario, el color y la presencia de portada y contraportada. Las consideraciones expuestas en la literatura sobre el tema llevan a plantear las hipótesis siguientes:

H1 El tamaño (dimensiones) del papel utilizado en el cuestionario ejerce una gran influencia en la tasa y en la calidad de la respuesta a encuestas postales: 1. Aunque un cuestionario con un gran tamaño puede generar en el entrevistado la sensación de que será largo de completar, la magnitud de la letra utilizada, los espacios disponibles, etc. hacen mucho más fácil su respuesta, lo que se traduce, en definitiva, en una tasa más elevada de respuesta.

2. La claridad y la amplitud de un cuestionario de un tamaño "grande» contribuye notablemente a conseguir una mayor calidad de respuesta, sobre todo en los colectivos de edad más elevada (Dillman, 1991).

H2 En relación con el formato de cubierta y de contraportada, se plantea como hipótesis que los cuestionarios que no tienen preguntas en la portada ni en la contraportada presentan una mayor calidad y tasa de respuesta.

H3 Tal y como ha demostrado la literatura sobre el tema, un cuestionario de color aumenta significativamente la tasa y la calidad de respuesta, debido fundamentalmente al «toque de conspicuidad» que adquiere cuando este es colocado en una mesa entre un montón de papeles blancos.

H4 La combinación los factores anteriores — gran tamaño, color verde y portada - produce una tasa y una calidad de respuesta mayores que el cuestionario de menor tamaño, color blanco y sin portada, pese a generar un ligero aumento en el coste final del estudio por el incremento del gasto en el envío de los cuestionarios.

\section{Resultados}

Dos meses después del primer envío, se logra obtener 440 cuestionarios, lo que implica una tasa de respuesta - considerando la fórmula de la respuesta mínima (en adelante RR1) de la AAPOR - del 56,6\%. En la exposición de resultados, se diferenciará, por un lado, el impacto de los aspectos visuales en la tasa de respuesta (apartado 3.a) y, posteriormente, la calidad de las que se proporcionaron.

\subsection{Tasa de respuesta}

Las devoluciones y la pérdida de algunos cuestionarios obliga a analizar el porcentaje de respuesta considerando exactamente el número de ellos que llegaron a su destino. El porcentaje de cuestionarios respondidos, que se muestra en la columna de la derecha de la tabla 2 , indica una tasa ligeramente más elevada de 
Tabla 2. Respuesta según los aspectos visuales considerados

\begin{tabular}{|c|c|c|c|}
\hline & $\begin{array}{c}\text { Enviados } \\
\text { (y recibidos) }\end{array}$ & Respondidos & $\begin{array}{c}\text { Porcentaje de } \\
\text { respondidos } \\
\text { respecto a } \\
\text { enviados }\end{array}$ \\
\hline \multicolumn{4}{|c|}{ Tamaño (chi cuadradoa 0,440, signif. 0,507 ) } \\
\hline Pequeño & 392 & 224 & $57,1 \%$ \\
\hline Grande & 392 & 216 & $55,1 \%$ \\
\hline \multicolumn{4}{|c|}{ Portada (chi cuadrado 0,341 , signif. 0,559 ) } \\
\hline Portada & 392 & 212 & $54,1 \%$ \\
\hline No portada & 392 & 228 & $58,2 \%$ \\
\hline \multicolumn{4}{|c|}{ Color (chi cuadrado 3,185 , signif. 0,074 ) } \\
\hline Blanco & 392 & 206 & $52,6 \%$ \\
\hline Verde & 392 & 234 & $59,8 \%$ \\
\hline
\end{tabular}

a. Al ser tablas de $2 \times 2$, estos valores corresponden a la corrección de continuidad.

Fuente: elaboración propia.

respuesta entre los cuestionarios pequeños, los que no tienen portada y los de color verde, diferencia que no llega a ser significativa en ninguno de los casos.

Pudiera ser que la similitud en la respuesta analizando todos los elementos uno a uno cambiara sustancialmente cuando fueran considerados conjuntamente. La elaboración de un modelo de regresión logística ordinal considerando los aspectos visuales como elementos independientes y la rapidez de respuesta ${ }^{7}$ como dependiente proporciona coeficientes no significativos en todos los términos independientes (ver tabla 3, modelo 1). En un segundo momento, se elaboró un modelo considerando las interacciones de las variables del diseño (tamaño y diseño, tamaño y color, diseño y color), pero tampoco se localizaron relaciones significativas (modelo 2 de la tabla 3), lo que conduce a confirmar que los aspectos visuales no presentan ninguna relación con la mayor o menor tasa de respuesta.

\subsection{Calidad de respuesta}

La calidad de respuesta será evaluada considerando tres aspectos: el número de preguntas no respondidas, el número de contestaciones en las preguntas multirrespuesta y la presencia de determinados efectos de respuesta. Este análisis se centra en las diecinueve variables «sustantivas» de la investigación, no se

7. Considerando el número de días y los estímulos recibidos, según el modelo del TDM (Dillman, 2000):

Respuesta rápida: antes de 8 días. Reciben carta de presentación, cuestionario y un recordatorio telefónico tres días después.

Respuesta media: entre 9 y 16 días. Además de los estímulos del grupo anterior, reciben un recordatorio agradeciendo la respuesta siete días después de la recepción del cuestionario.

Respuesta tardía: entre el día 17 y el 34 (fecha en la que se cierra el campo). A los estímulos del grupo anterior, se añade la recepción de un segundo cuestionario insistiendo en la importancia de su respuesta (recibido el día 16). 
Tabla 3. Modelo multivariante de la influencia de los aspectos visuales del cuestionario en la rapidez de la respuesta (regresión logística ordinal)a

\begin{tabular}{|c|c|c|c|c|}
\hline & \multicolumn{2}{|c|}{$\begin{array}{l}\text { Modelo 1: Considerando } \\
\text { solo los aspectos visuales } \\
\text { del cuestionario }\end{array}$} & \multicolumn{2}{|c|}{$\begin{array}{c}\text { Modelo 2: Aspectos } \\
\text { visuales e interacciones }\end{array}$} \\
\hline & Coeficientes & Wald & Coeficientes & Wald \\
\hline Tamaño (ref: grande) & $-0,281$ & 2,434 & $-0,333$ & 1,195 \\
\hline Portada (ref.: no portada) & $-0,017$ & 0,009 & 0,043 & 0,020 \\
\hline Color (ref: verde) & $-0,176$ & 0,949 & $-0,019$ & 0,004 \\
\hline Tamaño y portada (pequeño y portada) & & 0,157 & 0,189 & \\
\hline Tamaño y color (pequeño y blanco) & & & $-0,041$ & 0,013 \\
\hline Portada y color (portada y blanco) & & & $-0,298$ & 0,678 \\
\hline \multicolumn{5}{|l|}{ Rapidez en la respuesta } \\
\hline Rápida (0 = tardía) & $-0,263$ & 2,160 & $-0,225$ & 0,936 \\
\hline Media $(0$ = tardía) & 0,784 & $18,315^{\star \star}$ & $0,823^{\star \star}$ & 12,139 \\
\hline$R^{2}$ (Nagelkerte) & 0,009 & & 0,011 & \\
\hline$N$ & 440 & & 440 & \\
\hline
\end{tabular}

a. En la presentación de resultados, se han seguido las recomendaciones de Nicol y Pexman (1999).

b. Recomendamos precaución en la interpretación de las interacciones, porque el tamaño muestral de la investigación hace suponer que algunos coeficientes pueden haberse calculado con un escaso número de entrevistados.

** Significación al 0,01.

Fuente: elaboración propia.

consideran las siete preguntas que recogen la información sociodemográfica ni los datos de identificación del encuestado, fundamentalmente porque la mayor parte ha sido correctamente respondida. ${ }^{8}$ Tampoco se considera el número de preguntas de respuesta única que reciben más de una, por el escaso número de veces que esto sucede. La experiencia en investigaciones anteriores (Díaz de Rada, 1999; Díaz de Rada y Eguaras, 1999; Díaz de Rada, 2000; Díaz de Rada, 2001) recomendó acompañar cada pregunta con una frase entre paréntesis, advirtiendo que únicamente debía darse una contestación. Proceder de esta forma explica, sin duda, el bajo número de dobles respuestas.

Centrados en el primer aspecto, la cifra media de no respuestas por entrevistado es 27 . En contra de nuestras expectativas, la no respuesta es ligeramente más alta en los cuestionarios grandes y con portada, aunque la diferencia de medias es tan baja ${ }^{9}$ que no llega a ser significativa, lo que

8. Un 14\% de los entrevistados dejaron sin responder una pregunta, y 1,3\% dos y tres.

9. Número de preguntas no respondidas:

$\begin{array}{lll}\text { Diseño } & \text { Portada } & 26,9 \\ & \text { No portada } & 27,1 \\ \text { Tamaño } & \text { Pequeño } & 27,0 \\ & \text { Grande } & 27,1 \\ \text { Color } & \text { Blanco } & 27,2 \\ & \text { Verde } & 26,9\end{array}$


Tabla 4. Modelo multivariante de la influencia de los aspectos visuales del cuestionario en el número de preguntas no respondidas (regresión logística ordinal)

\begin{tabular}{|c|c|c|c|c|}
\hline & \multicolumn{2}{|c|}{$\begin{array}{l}\text { Modelo 1: Considerando } \\
\text { solo los aspectos visuales } \\
\text { del cuestionario }\end{array}$} & \multicolumn{2}{|c|}{$\begin{array}{l}\text { Modelo 2: Aspectos } \\
\text { visuales + rasgos } \\
\text { sociodemográficos }\end{array}$} \\
\hline & Coeficientes & Wald & Coeficientes & Wald \\
\hline Tamaño (ref.: grande) & 0,046 & 0,067 & 0,125 & 0,402 \\
\hline Portada (ref.: no portada) & 0,0005 & 0,000 & $-0,228$ & 1,296 \\
\hline Color (ref.: verde) & 0,162 & 0,824 & 0,208 & 1,121 \\
\hline Sexo (ref.: mujer) & & & $-0,342$ & $-2,434$ \\
\hline Edad & & & 0,020 & $5,316^{\star *}$ \\
\hline \multicolumn{5}{|l|}{ Nivel educativo (ref.: universitarios) } \\
\hline Sin terminar y primarios & & & $0,878^{\star \star}$ & 10,588 \\
\hline Secundarios & & & 0,360 & 1,898 \\
\hline \multicolumn{5}{|l|}{$\begin{array}{l}\text { Relación con la actividad } \\
\text { (ref.: resto no activos) }\end{array}$} \\
\hline Población activa (trabajadores y parados) & & & $-0,356$ & 1,769 \\
\hline Jubilados (han trabajado) & & & $-0,320$ & 0,729 \\
\hline \multicolumn{5}{|l|}{ Falta de respuesta } \\
\hline Baja $(0=$ alta $)$ & 0,975 & $28,110^{\star \star \star}$ & $-0,136$ & 0,075 \\
\hline Media $(0=$ alta $)$ & 0,320 & $3,237^{*}$ & 1,332 & $7,107^{\star \star}$ \\
\hline$\overline{R^{2} \text { (Nagelkerte) }}$ & & 0,002 & 0,106 & \\
\hline$N$ & & 440 & 380 & \\
\hline
\end{tabular}

* Significación al 0,10.

** Significación al 0,05.

*** Significación al 0,01.

Fuente: elaboración propia.

indica que estos aspectos visuales del cuestionario no afectan al número de preguntas no respondidas.

Pudiera ser que la escasa variación que presenta cada uno de estos aspectos visuales, considerados individualmente, cambiara si se consideraran conjuntamente, fundamentalmente por la presencia de interacciones cuando se combinan los diversos elementos (por ejemplo: cuestionario grande, con portada y verde). La elaboración de un modelo de regresión logística ordinal, considerando los aspectos visuales como elementos independientes y el número de no respuestas como dependiente, proporciona coeficientes no significativos en todos los términos independientes, lo que lleva a confirmar que los aspectos visuales tratados no presentan ninguna relación con la calidad de respuesta (modelo 1 de la tabla 4). Cuando, en este modelo, se introducen los rasgos sociodemográficos, siguiendo la metodología empleada en investigaciones similares (entre otras: Israel y Lamm, 2012; Lessler et al., 2012; Messer et al., 2012; Millar y Dillman, 2012), se produce un descenso significativo de todos los coeficientes relacionados con los aspectos visuales del cuestionario (modelo 2 de la tabla 4). El análisis de los coeficientes desvela que el aspecto que más influye en 
la calidad del cuestionario es la edad y el nivel de estudios. La primera muestra que el número de no respuestas crece a medida que aumenta la edad del entrevistado, y la segunda desvela que el número deno respuestas disminuye en los entrevistados con estudios universitarios. Pudiera ser que alguna de estas interactuara con algunos de los aspectos contemplados en el diseño, por lo que se llevó a cabo una tercera regresión añadiendo como variables independientes la interacción entre tamaño y edad, diseño y edad, color y edad, y lo mismo con el nivel de estudios. También se añadió la interacción entre edad y nivel de estudios, por si el hecho de combinarlas pudiera tener algún efecto no detectado. Pese a las limitaciones derivadas del tamaño muestral, ninguna de ellas aporta relación significativa, y ha sido el bajo número de unidades muestrales consideradas en cada combinación (y su efecto en los intervalos de confianza) lo que nos ha llevado a no presentarlos en la tabla 4. Un cuarto modelo, considerando únicamente las interacciones de las variables del diseño (tamaño y diseño, tamaño y color, diseño y color) tampoco presentó términos significativos. No han sido mostrados por lo mismo que el modelo anterior.

El segundo indicador de calidad —el número de respuestas en las preguntas multirrespuesta- será analizado diferenciando, por un lado, las tres preguntas que no presentan ninguna limitación al número de respuestas ${ }^{10} \mathrm{y}$, por otro, una pregunta que limita la respuesta a dos opciones. ${ }^{11}$ Dentro de las primeras, podría establecerse una segunda diferenciación atendiendo al número de categorías de respuesta disponibles (mostradas) en el cuestionario, en la medida en que estas pueden "animar» al entrevistado a contestar más (Dillman et al., 2009; Stern et al., 2012). La pregunta sobre compra emocional o por impulso permite doce respuestas; la pregunta sobre la posesión de tarjetas de "plástico» presenta diez posibilidades, y la que se refiere al conocimiento de organismos defensores de los consumidores, cuatro. Veamos cada una de ellas en detalle.

La pregunta con mayor número de opciones de respuesta plantea a los entrevistados sobre «el tipo de artículos que no puede evitar comprar cuando los ve». Se trata de una pregunta empleada para definir la compra por impulso (Luna Arocas et al., 1998), utilizada en numerosas ocasiones y que, en un estudio realizado en Valencia, desveló que un $47 \%$ de los entrevistados declararon una «inevitabilidad» de adquirir determinados productos (Luna Arocas y Fierres Giménez, 1998: 40). La utilización de esta pregunta en la realidad navarra desvela que la cantidad máxima de respuestas proporcionadas son siete (señaladas, concretamente, por dos personas). Un $20 \%$ de los entrevistados da una respuesta; un $8 \%$, dos, y otro $9 \%$, tres. La información más importante, considerando que la encuesta por correo suele detectar mayores valores de conductas desaprobadas socialmente (al ser la modalidad menos afectada por el efecto de deseabilidad

10. Artículos que no puede evitar comprar cuando los ve (pregunta 13), posesión de tarjetas de plástico a su nombre (pregunta 16) y conocimiento de organismos navarros defensores de los derechos de los consumidores (pregunta 19).

11. Relativa a los dos usos principales que da a las tarjetas que posee a su nombre (pregunta 16a). 
Tabla 5. Media de respuestas proporcionadas por las preguntas multirrespuesta

\begin{tabular}{lccc}
\hline & $\begin{array}{c}\text { Pregunta 13: Cosas que } \\
\text { compra siempre que ve } \\
\text { (12 respuestas máximo) }\end{array}$ & $\begin{array}{c}\text { Pregunta 16: Tarjetas } \\
\text { a su nombre (10 } \\
\text { respuestas máximo) }\end{array}$ & $\begin{array}{c}\text { Uso principal que } \\
\text { da a las tarjetas (2 } \\
\text { respuestas máximo) }\end{array}$ \\
\hline $\begin{array}{l}\text { Tamaño } \\
\text { Pequeño }\end{array}$ & 1,04 & 1,93 & 1,47 \\
$\quad$ Grande & 0,91 & 2,00 & 1,36 \\
\hline Portada & & & \\
$\quad$ Portada & 1,06 & 2,00 & 1,47 \\
$\quad$ No portada & 0,90 & 1,94 & 1,37 \\
\hline Color & $*, 92$ & 1,88 & 1,50 \\
$\quad$ Blanco & $* 0,82$ & 1,34 \\
$\quad$ Verde & $* 1,11$ & 2,04 & 1,42 \\
\hline Media respuestas & 0,98 & 1,97 &
\end{tabular}

* Significación al 0,05.

** Significación al 0,01.

Fuente: elaboración propia.

social), es que algo más de la mitad de la población analizada (un 55\%) responde que «no puede evitar comprar ningún artículo», ausencia de comportamiento impulsivo. Esta distribución de frecuencias desvela que la media de respuestas proporcionadas es de 0,98 . Solo el color del cuestionario presenta una diferencia significativa (tabla 5).

Un análisis de regresión, considerando como término dependiente el número de respuestas de esta pregunta y como independientes los aspectos visuales y los rasgos sociodemográficos, tal y como se ha procedido anteriormente (tabla 4), desvela que únicamente el sexo y la edad presentan relación con el número de respuestas (tabla 6) ${ }^{12}$. Ambas variables están relacionadas negativamente, lo que indica que son los jóvenes y las mujeres los colectivos que realizan un mayor número de compras impulsivas.

El número de contestaciones aumenta considerablemente en la segunda pregunta multirrespuesta, referida a la posesión de tarjetas «de plástico», puesto que nadie declara no poseer ninguna. La media de respuestas proporcionadas por cada entrevistado es de 2, que aumenta únicamente entre los que han respondido el cuestionario verde (aunque sin mostrar una diferencia significativa). Un análisis de regresión indica asociación significativa con el nivel de estudios y el sexo, ambas con valores positivos (tabla 6).

La tercera de las preguntas sin limitación de contestaciones está referida al conocimiento de cuatro organismos defensores de los derechos de los consumi-

12. A diferencia de las tablas 2 y 4, donde se mostraban los coeficientes de todas las variables independientes. En este caso, se ha utilizado un método secuencial de selección de variables predictoras, con el fin de seleccionar un modelo parsimonioso que ajuste correctamente. Cea d'Ancona (2002: 108-119) presenta una visión detallada de los fundamentos de esta forma de proceder. 
Tabla 6. Modelo multivariante de la influencia de los aspectos visuales del cuestionario en el número de respuestas proporcionadas por las preguntas multirrespuesta (regresión múltiple de mínimos cuadrados)

\begin{tabular}{|c|c|c|c|c|c|c|c|c|}
\hline & \multicolumn{2}{|c|}{$\begin{array}{l}\text { Pregunta 13: } \\
\text { Cosas que compra } \\
\text { siempre que ve }\end{array}$} & \multicolumn{2}{|c|}{$\begin{array}{c}\text { Pregunta 16: } \\
\text { Tarjetas disponibles }\end{array}$} & \multicolumn{2}{|c|}{$\begin{array}{c}\text { Pregunta 19: } \\
\text { Conocimiento sobre los } \\
\text { organismos defensores } \\
\text { de los consumidores }\end{array}$} & \multicolumn{2}{|c|}{$\begin{array}{l}\text { Pregunta 16a: } \\
\text { Uso principal que } \\
\text { da a las tarjetas }\end{array}$} \\
\hline & Standarized b & (t) & Standarized b & (t) & Standarized b & (t) & Standarized b & $(\mathrm{t})$ \\
\hline Tamaño (0 = grande) &,-- &,-- &,-- &,-- &,-- &,-- &,-- &,-- \\
\hline Portada ( $0=$ no portada) &,-- &,-- &,-- &,-- & $0,091^{*}$ & 1,771 &,-- &,-- \\
\hline Color $(0=$ verde $)$ &,-- &,-- &,-- &,-- &,-- &,-- &,-- &,-- \\
\hline $\operatorname{Sexo}(0=$ mujer $)$ & $-0,135^{\star \star \star}$ & $-2,577$ & $0,120^{\star \star}$ & 2,427 &,-- &,-- &,-- &,-- \\
\hline Edad & $-0,131^{\star \star}$ & $-2,201$ &,-- &,-- &,-- &,-- &,-- &,-- \\
\hline Nivel educativo &,-- &,-- & $0,294^{\star \star \star}$ & 5,958 &,-- &,-- & $0,281^{\star \star}$ & 5,633 \\
\hline \multicolumn{9}{|l|}{$\begin{array}{l}\text { Relación con la actividad } \\
\text { (ref.: resto no activos) }\end{array}$} \\
\hline $\begin{array}{l}\text { Población activa } \\
\text { (trabajadores y parados) }\end{array}$ &,-- &,-- &,-- &,-- &,-- &,-- & $0,251^{\star \star}$ & 5,032 \\
\hline Jubilados &,-- &,-- &,-- &,-- &,-- &,-- &,-- &,-- \\
\hline$R^{2}$ & 0,045 & & 0,096 & & 0,008 & & 0,188 & \\
\hline$N$ & 376 & & 376 & & 376 & & 368 & \\
\hline
\end{tabular}

* Significación al 0,10.

** Significación al 0,05.

*** Significación al 0,01.

--- Coeficientes no significativos (véase la nota 12).

Fuente: elaboración propia.

dores y muestra una media de 1,4 respuestas, producida porque el $8 \%$ de los entrevistados no conoce ninguno de los señalados en la pregunta. La comparativa de medias, en este caso, desvela una ausencia de diferenciación en todos los aspectos visuales considerados (lo más significativo es el color, con una diferencia significativa del 0,10$)$. El análisis de regresión presenta una relación positiva con la utilización de portada, que indica un mayor número de respuestas en los cuestionarios con portada.

La última de las preguntas multirrespuesta limita las contestaciones a dos, entre las cinco categorías mostradas. El análisis de datos desvela que nadie ha realizado más de dos, con lo cual se logra una media de 1,4 por cada entrevistado (únicamente responden los que tienen tarjetas). De nuevo, tan solo el color presenta diferencias significativas, de modo que la cantidad de preguntas no respondidas aumenta en los cuestionarios de color verde. Los coeficientes de regresión (tabla 6) indican un mayor número de respuestas en las personas con un alto nivel educativo y en las que son activas (laboralmente hablando).

El tercer indicador de calidad se refiere a la presencia de efectos de respuesta, concretamente, primacía y recencia. Desde que Sayre y Rugg detectaran los primeros response effects, a mediados de la década de 1940, un considerable número de estudios ha revelado la existencia de cambios 
en las contestaciones a preguntas cerradas, producidos por la variación del orden en que las categorías de respuesta son presentadas (entre otros, Krosnick, 1991; Tourangeau y Rasinski, 1988). Según estos expertos, los efectos de respuesta dependen del orden en que son colocadas las categorías de respuesta y de si estas son presentadas en tarjetas o son leídas en voz alta a los entrevistados. Cuando los entrevistados las pueden leer - lo cual se produce en encuestas autocumplimentadas o presenciales con ayudas visuales (como tarjetas de respuesta) —, hay una tendencia a seleccionar las primeras de ellas, efecto primacía (Krosnick, 1991). Esto es debido a que la mente procesa la primera opción en el momento en que son ofrecidas las restantes, y a que las primeras alternativas provocan la creación de un marco cognitivo por el que van a ser juzgadas las opciones subsiguientes. El efecto recencia se define por la mayor elección de la última categoría de respuesta, y es habitual en la comunicación exclusivamente oral que se utiliza en las encuestas presenciales (sin tarjeta) y en las encuestas telefónicas. En este caso, al tratarse de una encuesta que es leída por el entrevistado, solo puede considerarse el efecto primacía.

Para conocer su presencia, se cambió el orden de las categorías de respuesta en el $50 \%$ de los cuestionarios, de modo que la mitad de la muestra respondió a un cuestionario con un determinado orden de categorías (digamos, por ejemplo, A, B y C), y la otra mitad, el orden contrario (C, B y A). Para facilitar su gestión, los cuestionarios fueron numerados e identificados con números pares e impares. ${ }^{13}$ Posteriormente, fueron adaptados a las normas de formato aquí consideradas (tamaño, color y portada), con el fin de que fueran distribuidos el mismo número de cuestionarios pares e impares.

Se planteaba como hipótesis que únicamente las preguntas con un elevado número de categorías de respuesta presentarán efectos primacía. Para detectarlo, se han seleccionado las primeras respuestas de cada entrevistado, diferenciando si era un cuestionario impar o par, y, posteriormente, se ha promediado entre el total de personas que lo han respondido. Se explicará, a modo de ejemplo, la forma como se ha procedido con las preguntas con cuatro categorías, y se operará de la misma forma con el resto de categorías. Los 382 entrevistados que responden el cuestionario $\mathrm{A}, \mathrm{B}$ y $\mathrm{C}$ realizaron 302 elecciones

13. Véase, a modo de ejemplo, la diferencia en una de las preguntas del cuestionario.

Cuestionarios impares:

03. Según su punto de vista, ¿qué cree que le produce a la gente más satisfacción cuando compra algún objeto? (Marcar una respuesta)

- El acto de compra.

- La posesión del producto.

— La utilidad que se puede obtener del producto comprado.

Cuestionarios pares:

03. Según su punto de vista, ¿qué cree que le produce a la gente más satisfacción cuando compra algún objeto? (Marcar una respuesta)

- La utilidad que se puede obtener del producto comprado.

- La posesión del producto.

- El acto de compra. 
Tabla 7. Efecto primacía: número medio de elecciones de la primera categoría de respuesta (sin considerar las preguntas dicotómicas)

\begin{tabular}{lcccc}
\hline & \multicolumn{4}{c}{ Número de categorías de las preguntas: } \\
& (7 preguntas) & $\begin{array}{c}\text { Cuatro } \\
\text { (5 preguntas) }\end{array}$ & $\begin{array}{c}\text { Cinco } \\
\text { (3 preguntas) }\end{array}$ & $\begin{array}{c}\text { Seis y siete } \\
\text { (2 preguntas) }\end{array}$ \\
\hline $\begin{array}{l}\text { Tamaño } \\
\text { Pequeño }\end{array}$ & 0,18 & 0,07 & 0,12 & 0,12 \\
$\quad$ Grade & 0,17 & 0,06 & 0,12 & 0,11 \\
\hline $\begin{array}{l}\text { Portada } \\
\text { Portada }\end{array}$ & 0,17 & 0,06 & 0,11 & 0,11 \\
$\quad$ No portada & 0,18 & 0,06 & 0,12 & 0,12 \\
\hline $\begin{array}{l}\text { Color } \\
\quad \text { Blanco }\end{array}$ & & & & \\
$\quad$ Verde & 0,18 & 0,06 & 0,11 & 0,10 \\
\hline
\end{tabular}

* Significación al 0,05.

** Significación al 0,01.

Fuente: elaboración propia.

de la primera categoría de respuesta (A) en estas preguntas, que, considerando que hay cinco preguntas con este número de categorías, supone un promedio de 60,4 elecciones por cada pregunta (302/5). Cuando se tiene en cuenta el número de entrevistados que responden este cuestionario (382), se obtiene un «indicador de primacía» de 0,16 (60,4/382). En el cuestionario C B y A, son 372 las elecciones de la primera categoría, que, divididas entre las cinco preguntas con cuatro categorías, proporciona un valor de 74,4 elecciones por pregunta (372/5). Estos cuestionarios son respondidos por 392 personas, lo que supone un indicador de primacía de 0,19 (74,43/392).

No hay diferencias en ninguna de las medias mostradas en la tabla 7 , lo que impide aceptar la hipótesis planteada, puesto que las preguntas con cinco y seis categorías presentan un efecto primacía muy similar a las que tienen tres categorías. Resalta la baja presencia de efecto primacía en las preguntas con cuatro categorías de respuesta. El interior de la tabla tampoco proporciona evidencias de que la utilización de un diseño de cuestionario u otro genere más o menos efecto primacía.

\section{Conclusiones}

El fin de este trabajo ha sido conocer la influencia de tres aspectos visuales del cuestionario en la tasa y calidad de respuesta, uno de los aspectos menos estudiados en la investigación mediante encuesta. Basados en la investigación internacional que da cuentas de diferencias según las dimensiones del cuestionario, el color y el formato de cubierta, en este trabajo, se ha analizado la influencia de dos tamaños $(14,85 \mathrm{~cm} \times 21 \mathrm{~cm}$ y $21 \mathrm{~cm} \times 29,7 \mathrm{~cm})$, dos colores (verde y blanco) y dos formatos de cubierta (con portada o sin portada) en una encuesta postal realizada en España. 
Los resultados expuestos desvelan que ninguno de los elementos considerados afectan a la tasa y a la calidad de la respuesta, esta última calculada considerando el número de preguntas no respondidas, el número de respuestas proporcionadas por las preguntas multirrespuesta y la presencia del efecto primacía.

A la luz de estos hallazgos, aconsejamos utilizar el cuestionario de dimensiones $14,85 \mathrm{~cm} \times 21 \mathrm{~cm}$ blanco y sin portada, por razones económicas. Este cuestionario supone un ahorro de papel del 50\%, cuando se compara con el cuestionario grande y sin portada, y del 66\%, cuando se compara con el cuestionario grande y con portada, además de obtener una tasa de respuesta superior al resto, tal y como se aprecia en la tabla 2 . En cuanto a la calidad de la respuesta, este consigue un menor número de preguntas no respondidas, un mayor número de respuestas en las preguntas multirrespuesta (tabla 7) y un ligero mayor efecto primacía; diferencias que — en todos los casos - no llegan a ser significativas.

Es importante destacar las especificidades propias de nuestro país, en comparación con las investigaciones realizadas en EE. UU., cuando algunos expertos (entre otros, Dillman, 1991) han señalado que los cuestionarios de reducidas dimensiones son respondidos mejor por los jóvenes, mientras que la población de más edad responde mejor cuestionarios de mayor tamaño. En el apartado 3.a, se ha indicado la ausencia de relación entre los aspectos visuales y la calidad de la respuesta; en el 3.b, la relación con la tasa de respuesta, no habiéndose localizado diferencias significativas con los rasgos sociodemográficos de los entrevistados (tablas 4 y 6 ).

Conviene precisar que la conclusión de naturaleza metodológica más importante que se deriva de esta investigación es que las variables que se están manejando - en cuanto a los efectos del diseño del cuestionario en la tasa de respuesta - son insuficientes. La literatura específica parece depositar más confianza en la dimensión que en el color, cuando nosotros hemos encontrado lo contrario. En las tablas, se observan, además, algunas tendencias contradictorias que no son fáciles de explicar desde el modelo teórico más sólido con el que contamos hasta el momento: el TDM de Dillman. Ello sugiere que es importante realizar un esfuerzo para identificar las variables intermedias que relaciona el diseño del cuestionario con los comportamientos de respuesta. Muy posiblemente, estas variables cuenten con valores diferentes según la procedencia de las muestras, por lo que, tal vez, los estudios metaanalíticos no nos puedan suministrar resultados prometedores a día de hoy.

\section{Referencias bibliográficas}

Avdeyeva Olga, Ana y Matland, Richard E. (2013). «An Experimental Test of Mail Surveys as a Tool for Social Inquiry in Russia». International Journal of Public Opinion Research [en línea], 25 (2), 173-194. $<$ http://dx.doi.org/10.1093/ijpor/eds020>.

Ballina Ballina, Francisco Javier de la (1988). «La encuesta postal en la investigación de mercados». Investigación y Marketing, 58, 59-64. 
Benthlehem, Jelke; Cobben, Fannie y Schouten, Barry (2011). Handbook of nonresponse in household surveys [en línea]. Nueva York: Wiley. <http://dx.doi.org/10.1002/9780470891056>.

Blom, Annelies G.; Lynn, Peter y Jäckle, Annette (2008). «Understanding cross-national differences in unit non-response: The role of contact data». ISER Working Papers Series [en línea], 2008-01, noviembre. Colchester: University of Esse.

<http://www.iser.essex.ac.uk/publications/working-papers/iser/2008-01> [Consulta: 11 junio 2011].

Bradley, Nigel (1999). «Sampling for Internet Surveys: An Examination of respondent Selection for Internet Research». International Journal of Market Research, 41, 387-395.

Brick, Michael J.; Montaquila, Jill M.; Han, Daifeng y Williams, Douglas (2012). «Improving Response Rates for Spanish Speakers in Two-Phase Mail Surveys». Public Opinion Quarterly, 76, 733-766.

Cea d’Ancona, María Ángeles (2002). Análisis Multivariable. Teoría y práctica en la investigación social. Madrid: Síntesis.

Cook, Colleen; Heath, Fred y Thompson, Russel L. (2000). «A Meta-Analysis of Response Rates in Web- or Internet Based Surveys». Educational and Psychological Measurement [en línea], 60 (6), 821-836. <http://dx.doi.org/10.1177/00131640021970934>.

Couper, Mick P. (2005). "Technology Trends in Survey Data Collection». Social Science Computer Review [en línea], 23 (4), 486-501. <http://dx.doi.org/10.1177/0894439305278972>.

- (2011). «The Future of Modes of Data Collection». Public Opinion Quarterly [en línea], 75, 889-908. <http://dx.doi.org/10.1093/poq/nfr046>.

Czaja, Ronald F. y Blair, Johnny (1996). Designing Surveys. California: Pine Forge.

De Leeuw, Edith D. (1992). Data Quality in Mail, Telephone and Face to Face Surveys. Amsterdam: TT-Publikaties.

De Leeuw, Edith D.; Hox, Joop J. y Dillman, Don A. (2008). «Mixed mode surveys: When and why». En: De Leeuw, Edith D.; Hox, Joop J. y Dillman, Don A. (eds.). International Handbook of Survey Methodology. Nueva York: Lawrence Erlbaum Associates and European Association of Methodology.

DíAz DE RADA, Vidal (1999). "Viejos tópicos y nuevas realidades en la encuesta postal». Metodología de Encuestas, 1 (1), 107-125.

- (2000). «Using Dillman's Total Design Method (TDM) in a South Europe country: Spain». Ponencia presentada en WAPOR Annual Conference, Portland, Oregon, 17 y 18 de mayo.

- (2001). «Mail Surveys Using Dillman’s TDM in a Southern European Country: Spain». International Journal of Public Opinion Research, 13 (2), 159-172.

DíAz de RADA, Vidal y Andreu, Jaime (2004). «Resulta apropiado utilizar la guía de teléfonos como marco muestral en sondeos?». Revista Española de Investigaciones Sociológicas (ISSN 0210-5233) [en línea], 108, 123-149. (Tipo de publicación A, código UNESCO: 630201, 630303). <http://dx.doi.org/10.1093/ijpor/13.2.159>.

Díaz de Rada, Vidal y Eguaras, C. (1999). Atitudes y opiniones del socio del Club Atlético Osasuna. Trabajo sin publicar.

Dillman, Don A. (1978). Mail and Telephone Surveys. Nueva York: Willey. 
- (1991). "The Design and Administration of Mail Surveys». Annual Review of Sociology [en línea], 17, 225-249. <http://dx.doi.org/10.1146/annurev.so.17.080191.001301>.

- (2000). Mail and Internet Surveys: The Tailored Design Method. 2. ${ }^{\mathrm{a}}$ ed. Nueva York: Wiley.

Dillman, Joan J. y Dillman, Don A. (1995). «The influence of questionnaire cover design on response to mail surveys». Presentation to the International Conference on Survey Measurement and Process Quality. Bristol, Reino Unido, 109-114.

Dillman, Don A.; Smyth, Jolene D. y Christian, Leah Melanie (2009). Internet, Mail and Mixed-Mode Surveys: The Tailored Design Method. 3. ${ }^{\mathrm{a}}$ ed. Nueva York: John Wiley.

Fox, Richard J.; Crask, Melvin R. y Jonghoon Kim, Ph. D. (1988). «Mail Survey Response Rate (A Meta-analysis of Selected Techniques for Inducing Response)». Public Opinion Quarterly [en línea], 52, 467-491. <http://dx.doi.org/10.1086/269125>.

Graetz, Brian (1985). «The potential of mail surveys». Australian and New Zealand Journal of Sociology, 21, 445-455. <http://dx.doi.org/10.1177/144078338502100308>.

Groves, Robert M.; Presser, Singer y Dipko, Son (2004). «The Role of Topic Interest in Survey Participation Decisions». Public Opinion Quarterly [en línea], 68 (1), 2-31. <http://dx.doi.org/10.1093/poq/nfh002>.

Heerwegh, Dirk (2009). "Mode differences between face-to-face and web surveys: An experimental investigation of data quality and social desirability effects». International Journal of Public Opinion Research [en línea], 21, 111-120. $<$ http://dx.doi.org/10.1093/ijpor/edn054>.

Heerwegh, Dirk y Loosveldt, Geert (2008). «Explaining the intention to participate in a web survey: A test of the theory of planned behavior». International Journal of Social Research Methodology, 12, 181-195.

Holmberg, Anders; Lorenc, Boris y Werner, Peter (2010). «Contact Strategies to improve participation via the Web in a mixed-mode mail and web surveys». Journal of Official Statistics, 26 (3), 465-480.

Israel, Glenn D. y Lamm, Alexa J. (2012). «Item Non-Response in a Client Survey of the 2 General Public». Survey Practice [en línea], abril. <http://surveypractice.wordpress.com/2012/4> [Consulta: 15 noviembre 2012].

Jenkins, Cleo R. y Dillman, Don A. (1997). «Towards a Theory of Self-Administered Questionnaire Design». En: Lyberg, Lars et al. (ed.). Survey Measurement and Process Quality. Nueva York: John Wiley and Sons.

Jobber, David y Sanderson, Stuart (1983). «The Effects of a Prior Letter and Colored Questionnaires Paper on Mail Survey Response Rates». Journal of the Market Research Society, 25, 339-349.

Johnson, Timothy P.; Parsons, Jennifer A.; Warnecke, Richard B. y Kaluny, Arnold D. (1993). "Dimensions of Mail Questionnaires and Response Quality». Sociological Focus [en línea], 26, 271-274. <http://dx.doi.org/10.1080/00380237.1993.10570625>.

Jussaume, Raymond A. y Yamada, Yoshiharu (1990). "A Comparison of the Viability of Mail Surveys in Japan and the United States». Public Opinion Quarterly [en línea], 54, 219-228. <http://dx.doi.org/10.1086/269199>. 
Kaplowitz, Michael D.; Hadlock, Timothy D. y Levine, Ralph (2004). «A Comparison of Web and Mail Survey Response Rates». Public Opinion Quarterly [en línea], 68, 94-101. <http://dx.doi.org/10.1093/poq/nfh006>.

KeEter, Scott (1995). «Estimating telephone noncoverage bias with a telephone survey». Public Opinion Quarterly [en línea], 59, 196-217. <http://dx.doi.org/10.1086/269469>.

Krosnick, Jon A. (1991). "Response Strategies for Coping with the Cognitive Demands of Attitude Measures in Surveys». Applied Cognitive Psychology [en línea], 5, 213-236. <http://dx.doi.org/10.1002/acp.2350050305>.

Lessler, Virginia M.; Newton, Lydia y Yang, Daniel (2012). «Item Non-Response in Web and Mail Responses to General Public Surveys». Survey Practice [en línea], abril. <http://surveypractice.wordpress.com/2012/4> [Consulta: 15 noviembre 2012].

Lozar Manfreda, Katja; BosnjaK, Michael; BerzelaK, Jerney; HaAs, Iris y Vehovar, Vasja (2008). «Web surveys versus other survey modes: A meta-analysis comparing response rates». International Journal of Market Research, 50 (1), 79-104.

Luna Arocas, Roberto y Fierres Giménez, Rosa (1998). «Incidencia de la compra por impulso en la ciudad de Valencia». Investigación y Marketing, 60, 36-44.

Luna Arocas, Roberto; Quintanilla, Ismael y Berenguer, Gloria (1998). La compra impulsiva y la compra patológica: El modelo CAC. IVIE. SERIE EC WPEC 98-11, 3-32.

Manheim, Jarold B. y Rich, Richard C. (1986). Empirical Political Analysis: Research Methods in Political Science. Nueva York: Longman. Versión española: Análisis político empírico: Métodos de investigación en Ciencia Política. Madrid: Alianza, 1988.

Matteson, Michael T. (1974). «Type of Transmittal Letter and Questionnaire Color as Two Variables Influencing Response Rates in a Mail Survey». Journal of Applied Psychology [en línea], 59, 535-536. <http://dx.doi.org/10.1037/h0037275>.

Medway, Rebecca L. y Fulton, Jenna (2012). «When more gets you less: A meta-analysis of the effects of concurrent web options on mail survey response rates». Public Opinion Quarterly, 76, 733-766.

Medway, Rebecca y Tourangeau, Roger (2011). An investigation of the impact of stylistic features on web survey responses [en línea]. Comunicación presentada en la $66^{\text {th }}$ Annual Conference of the American Association for Public Opinion Research, Phoeniz, AZ. <http://dx.doi.org/10.1093/poq/nfs047>.

Messer, Benjamin L. y Dillman, Don A. (2011). "Surveying the general public over the internet using address-based sampling and mail contact procedures». Public Opinion Quarterly [en línea], 75 (3), 429-457. Doi: 10.1093/poq/nfr021. <http://dx.doi.org/10.1093/poq/nfr021>.

Messer, Benjamin L.; Edwards, Michelle L. y Dillman, Don A. (2012). «Determinants of Item Nonresponse to Web and Mail Respondents in Three Address-Bases Mixed-Mode Surveys of the General Public». Survey Practice [en línea], 5 (22). $<$ http://surveypractice.wordpress.com/2012/4> [Consulta: 15 noviembre 2012].

Millar, Morgan M. y Dillman, Don A. (2012). "Do Mail and Internet Surveys Produce Different Item Nonresponse Rates». Survey Practice [en línea], 5 (2). $<$ http://surveypractice.wordpress.com/2012/4> [Consulta: 15 noviembre 2012]. Miquel, Salvador et al. (1999). Investigación de mercados. Madrid: McGraw Hill. 
Muñoz Leiva, Francisco; Sánchez Fernández, Juan; Montoro Ríos, Francisco e IbáÑeZ Zapata, José Ángel (2009). "Improving the response rate and quality in web-based surveys through the personalization and frequency of reminder mailings». Quality and Quantity [en línea], 44, 1037-1052. <http://dx.doi.org/10.1007/s11135-009-9256-5>.

Nederhof, Anton J. (1988). «Effects of a Final Telephone Reminder and Questionnaire Cover Design in Mail Surveys». Social Science Research [en línea], 17, 353-361. <http://dx.doi.org/10.1016/0049-089X(88)90008-7>.

Newman, Laurence W. (1997). Social Research Methods: Qualitative and Quantitative Approaches. Boston: Allyn y Bacon.

Nicol, Adelheid A.M. y Pexman, Penny M. (1999). Presenting your findings: A practical guide for creating tables. Washington: American Phychological Association

Pasadas del Amo, Sara y Trujillo Carmona, Manuel (2013). Afijación óptima basada en costes para muestras telefónicas recogidas en marcos duales. 1st Southern European Conference on Survey Methodology (SESM) y VI Congreso de Metodología de Encuestas. Barcelona, 12 a 14 de diciembre.

Petersen, Thomas (2008). «Split Ballots as an experimental approach to public opinion research». En: Donsbach, Wolfgang y Traugott, Michael W. (eds.). The SAGE Handbook of Public Opinion Research. Thousand-Oaks, LA: Sage.

Peytchev, Andy; Baxter, Rodney K. y Carley-Baxter, Lisa R. (2009). "Not All Survey Effort is Equal: Reduction of Nonresponse Bias and Nonresponse Error». Public Opinion Quarterly [en línea], 73, 785-806. <http://dx.doi.org/10.1093/poq/nfp037>.

Riba, Clara; Torcal, Mariano y Morales, Laura (2010). «Estrategias para aumentar la tasa de respuesta y los resultados de la Encuesta Social Europea en España». Revista Internacional de Sociología [en línea], 68, 603-635. <http://dx.doi.org/10.3989/ris.2008.12.17>.

Sánchez, María Elena (1992). «Effects of questionnaire design on the quality of survey data». Public Opinion Quarterly [en línea], 56, 206-217. <http://dx.doi.org/10.1086/269311>.

Sarantakos, Sotirios (2003). Social research. Londres: MacMillan.

Schuman, Howard y Presser, Stanley (1981). Questions and answer in attitude surveys: Experiments on question form, wording and context. Nueva York: Academic.

Sheastley, Paul B. (1983). "Questionnaire construction and item writing». En: Rossi, Peter H.; Wright, James D. y Anderson, Andy B. (eds.). Handbook of Survey Research. San Diego: Academic Press, 159-230.

Shin, Tse Hua y FAn, Xitao (2008). «Comparing Response Rates from Web and Mail Surveys: A Meta-Analysis». Field Methods [en línea], 20, 249-271. <http://dx.doi.org/10.1177/1525822X08317085>.

Smyth, Jolene y Olson, Kristen (2011). Comparing numeric and text open-end responses in mail and web surveys [en línea]. Comunicación presentada en la $66^{\text {th }}$ Annual Conference of the American Association for Public Opinion Research, Phoeniz, AZ.

Stern, Michael J.; Smyth, Jolene D. y Mendez, Jeanette (2012). «The Effects of Item Saliency and Question Design on Measurement Error in a Self-Administered Survey». Field Methods [en línea], 24 (1), 3-27. <http://dx.doi.org/10.1177/1525822X11419478>.

Stoop, Inkele; Billiet, Jaak; Koch, Achim y Fitzgerald, Rory (2012). Improving survey response: Lessons learned from the European Social Survey. Chichester (West Sussex): Wiley. 
Sudman, Stanley y Bradburn, Norman (1987). Asking Questions. San Francisco: Jossey Bass.

Sun, Hanyu (2011). Investigating the impact of scale height, width and spacing on responses in web surveys. Comunicación presentada en la $66^{\text {th }}$ Annual Conference of the American Association for Public Opinion Research, Phoeniz, AZ.

Tourangeau, Roger; Couper, Mick P. y Conrad, Frederick (2004). «Spacing, position and order: Interpretive heuristic for visual features of survey questions». Public Opinion Quarterly [en línea], 68, 368-393. <http://dx.doi.org/10.1093/poq/nfh035>.

Tourangeau, Roger y Rasinski, Kenneth (1988). "Cognitive Processes Underlying Context Effects in Attitude Measurement». Psychological Bulletin [en línea], 103, 299-314. <http://dx.doi.org/10.1037/0033-2909.103.3.299>.

Tucker, C.; Lepkowski, J. M.; Casady, R. J. y Groves, R. M. (1992). «Commercial Residential Telephone Lists: Their characteristics and uses in survey design». Social Science Computer Review [en línea], 10, 158-172.

Visauta, Remigio (1989). Técnicas de Investigación Social I: Recogida de datos. Barcelona: PPU. 


\section{Anexo 1: Cuestionario utilizado ${ }^{14}$}

01. ¿Con qué frecuencia se detiene a ver escaparates? (Marcar una respuesta) - Casi todos los días.

- De vez en cuando, cuando ve algo que le llama la atención.

- Cuando va a comprar o cuando necesita alguna cosa.

- Nunca.

02. ¿Usted podría decir que disfruta cuando compra algún objeto o, por el contrario, es algo que tiene que hacer porque lo necesita y no le queda otro remedio? (Marcar una respuesta)

- Disfruta comprando.

- Le resulta indiferente. No le produce placer ni desagrado.

- Sólo va a comprar cuando no le queda más remedio.

03. Según su punto de vista, ¿qué cree que le produce a la gente más satisfacción cuando compra algún objeto? (Marcar una respuesta)

- El acto de compra.

— La posesión del producto.

— La utilidad que se puede obtener del producto comprado.

04.Y a usted, ¿que le produce más satisfacción cuando compra algún objeto? (Marcar una respuesta)

- El acto de compra.

- La posesión del producto.

— La utilidad que se puede obtener del producto comprado.

05. ¿Cómo suele realizarse la compra de productos de alimentación y droguería para su hogar? (aunque no sea usted quien la realice) (Marcar una respuesta) - Compra todos o casi todos los días.

- Suele comprar cada dos o tres días.

- Compra fundamentalmente un día a la semana, aunque a diario adquiera cosas sueltas.

- Realiza una gran compra mensual, aunque haga pequeñas compras a lo largo del mes.

06. ¿Suele hacer personalmente la compra de productos de alimentos y droguería para su hogar? (Marcar una respuesta)

- Siempre.

- Casi siempre.

- Alguna vez.

- Rara vez.

- Nunca. (Ir a pregunta 7)

14. Es importante tener presentes las variaciones de formato (tamaño, color y presencia o ausencia de portada y/o contraportada) mostradas en la tabla 1 . No se reproduce la portada porque se ha mostrado en la figura 1. 
6a. Y de los siguientes tipos de establecimientos, ¿dónde realiza la mayor parte de estas compras? (Marcar una respuesta)

- Tienda tradicional.

- Supermercado (o maxisúper).

- Hipermercado.

- Mercado de abastos.

- Grandes almacenes.

- Centro comercial.

- Otros:

07. La compra de ropa y calzado para usted, ¿suele realizarla personalmente, o deja que otra persona la haga por usted? (Marcar una respuesta)

- Suele ir de compras personalmente.

— Deja que sea otra persona quien la haga. (Ir a pregunta 8).

7a. Y de los siguientes tipos de establecimientos, ¿dónde realiza la mayor parte de estas compras? (Marcar una respuesta)

- Tienda tradicional.

- Supermercado (o maxisúper).

- Hipermercado.

- Grandes almacenes.

- Centro comercial.

- Otros:

08. Preferentemente, ¿cuándo suele comprar la ropa y el calzado para usted o sus familiares? (Marcar una respuesta)

- Al comienzo de cada temporada.

- Cuando realmente necesita algo.

- Cuando ve algo que le gusta.

09. Cuando deja de usar prendas, ¿̇suele ser porque están en mal estado, porque ya no le gustan o porque se han pasado de moda? (Marcar una respuesta)

- Están en mal estado.

- Ya no me gustan.

— Se han pasado de moda.

10. ¿Suele comprar cosas que luego no usa? (Marcar una respuesta)

- Sí, frecuentemente.

- Alguna que otra vez.

- No, nunca.

11. ¿Diría usted que, en determinados productos (pantalones, etc.), suele utilizar siempre la misma marca o, por el contrario, cambia con frecuencia? (Marcar una respuesta)

- Suele utilizar siempre las mismas marcas.

- No se fija en la marca.

- Suele cambiar de marca con frecuencia. 
12. Voy a citarle diversos motivos por los que la gente compra «marcas prestigiosas", ¿podría decirme si está de acuerdo o en desacuerdo con cada uno? (Para cada acción, marcar si se está de acuerdo o en desacuerdo)

Respuestas

La gente compra marcas "prestigiosas»:

- Para presumir

Acuerdo

Desacuerdo

- Porque se fía más de productos de marcas conocidas Acuerdo

Desacuerdo

- Por la calidad y la garantía de la marca Acuerdo

Desacuerdo

- Porque hay marcas que proporcionan una cierta «individualidad», van con la forma de ser de cada uno
Acuerdo
Desacuerdo

- Porque se encuentra más cómoda con esas marcas Acuerdo

Desacuerdo

- Para diferenciarse de otras personas
Acuerdo
Desacuerdo

- Porque, al comprar productos con marcas conocidas, se puede realizar la compra en menos tiempo Acuerdo

Desacuerdo

13. ¿Qué cosas no puede evitar comprar cuando las ve? (Marcar todas las que se deseen)

- Ropa.

- Calzado.

- Artículos de deporte.

- Perfumería/cosméticos.

- Libros.

- Música.

- Ninguna.

- Herramientas.

- Muebles.

- Electrodomésticos.

- Informática.

- ¿Alguna otra?

- Artículos para el coche.

14. Centrándonos en la ropa y en el calzado, ¿cómo cree usted que son los productos que se venden en las rebajas? (Marcar una respuesta)

- Suelen ser productos de calidad rebajados de precio.

- Son productos un poco pasados de moda.

- La mayor parte de los productos son auténticos engaños.

15. ¿¿Suele comprar productos textiles que están de oferta o rebajados? (Marcar una respuesta)

- Muy frecuentemente.

- Frecuentemente.

- Rara vez.

- Nunca. (Ir a pregunta 16) 
15a. De las veces que compra en «temporada de rebajas» (rebajas de enero, etc.), ¿suele adquirir productos que no necesita pero que le vendrán muy bien en un futuro? (Marcar una respuesta)

— La gran mayoría de los productos que compra en rebajas están influidos por esa idea.

- Compra bastantes productos (con esa idea).

- Compra pocos productos (con esa idea).

- Nunca compra productos pensando en su utilidad posterior.

16. De las siguientes tarjetas que voy a leerle, ¿podría decirme cuáles posee usted a su nombre? (Marcar todas las que se tengan)

- VISA. - Mastercard.

- VISA Electron. - Eurocard.

- 4B o Servired. - Tarjeta de compra de algún establecimiento (Corte Inglés, Unzu, Eroski, Cortefiel, etc.).

- Clave, Líder u otras 6000.

- Dinner's Club.

- American Express

- Ninguna. (Ir a pregunta 17)

- Otras:

16a. ¿Cuáles son los dos usos principales que da a esta tarjeta o tarjetas? (Marcar máximo dos respuestas)

- Comprar productos de precio elevado.

- Comprar cualquier tipo de productos.

- Sacar dinero en cajeros automáticos.

- Pagar servicios (viajes, restaurantes).

- Otros:

16b. ¿Alguna vez ha gastado con su tarjeta más dinero del que usted tenía? - Sí.

- No.

17. Cambiando de tema, ¿ha presentado alguna reclamación por no quedar satisfecho tras la compra de algún producto o servicio?

- Sí. (Ir a pregunta 19)

- No.

18. Y si tuviera que reclamar, ¿qué procedimiento utilizaría? (Marcar una respuesta)

- Hablaría con el vendedor o con la persona responsable.

- Reclamaría ante una institución pública.

- Reclamaría ante una asociación de consumidores.

- Denunciaría el hecho en los medios de comunicación.

— Lo denunciaría en el juzgado. 
19. En Navarra, existen varios organismos defensores de los derechos de los consumidores, ¿Conoce el nombre de alguno? (Marcar todos los que se conozcan)

- Oficinas municipales de información al consumidor (OMIC).

- Juntas arbitrales de consumo.

- Asociación de Consumidores Irache.

- Asociación de Amas de Casa Santa María La Real.

20. ¿Cuál es su relación con el cabeza de familia? (Considerando al cabeza de familia como la persona que aporta más ingresos al hogar)

- Cabeza de familia.

- Esposa/o o compañera/o.

- Hijo/a.

- Padre, madre, suegro o suegra.

- Hermano/a o cuñado/a.

21. ¿Podría decirme cuál es su ocupación y la del cabeza de familia?

- Su ocupación:

- Cabeza de familia:

22. ¿Cuál es su nivel de estudios? Indicar los estudios acabados de mayor nivel. (Marcar una respuesta)

— Ningún estudio.

- Menos de estudios primarios (menos de 6 años en la escuela).

- Estudios primarios completos (certificado de escolaridad).

- Formación profesional, bachiller elemental, EGB.

- Bachiller superior, BUP, FP II.

- Estudios universitarios medios.

— Estudios universitarios superiores.

23. ¿Cuántas personas viven actualmente en su hogar, incluido usted? (Apuntar número)

24. ¿Cuál es su estado civil? (Marcar una respuesta)

- Soltero/a.

- Casado/a o convive con pareja.

- Separado/a o divorciado/a.

- Viudo/a.

25. ¿Podría decirme qué edad tiene usted?

26. Señale, por favor, si es hombre o mujer.

- Hombre.

- Mujer.

Muchas gracias por su colaboración 
Si hay algo que a usted le gustaría añadir acerca de sus hábitos de consumo o cualquier otro comentario sobre los temas tratados en este cuestionario, hágalo, por favor, en el espacio que sigue a continuación.

Agradecemos su ayuda y su esfuerzo al responder a este cuestionario. Si desea obtener un resumen de los resultados obtenidos, por favor, ponga su nombre y dirección en la solapa del sobre de respuesta (no en este cuestionario). Le enviaremos la información con mucho gusto. 